\title{
The inevitable march of intra oral digital scanning
}

\author{
Marcos White, practice owner and lead cosmetic dentist at The Courtyard Clinical Academy, talks \\ about the inevitable march of intra oral digital scanning
}

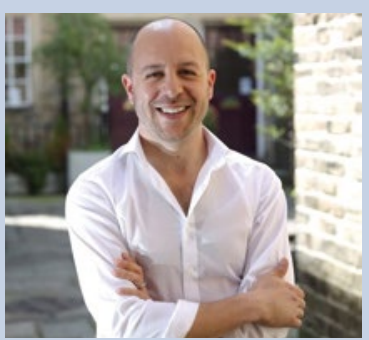

Marcos White
Since the advent of the iPhone, software and tech changed forever. They became intuitive. You did not need a handbook or an instruction manual. You just turned it on and figured it out. It also meant it became cool to be tech savvy. From that point on, it was mandatory and standard that any tech innovation had to deliver a direct improvement to your life immediately.

That same 'essence' of tech innovation has now hit dentistry with some clear similarities and some distinct differences. Digital intra oral scanners have been around for a while, but recently more and more of the UK dental community has begun to appreciate the accuracy, efficiency and cost savings they will bring to the profession. This will make them inevitable. It's not if, but when and which.

The tipping point is coming and just like the CDs replacing vinyl, an unstoppable change is coming. Are you ready?

Marcos White is a self-proclaimed early adopter. Winner of Most Innovative Practice at the Private Dentistry Awards he has always sought for new ways of doing old things. So it was with digital dentistry. Marcos invested in his first intra oral scanner in June 2013 to increase the accuracy of his complex restorative cases. Since then, he has owned and tested all of the three major scanning systems, but has settled on iTero and only uses these scanners throughout his solely digital practice.

In the last 12 months since buying three iTero's he has seen his revenue increase by a staggering $33 \%$. When asked if there were any other factors that could explain this, he states categorically no.

'Everything else is unchanged,' he says. 'It's the digital angle and you can only understand what I mean by that, if as a dentist, you appreciate that a scanner is not just a digital replacement for impression taking. It is a complete rethinking of the entire restorative process.

'From consultation, through consent and digitally simulated outcomes, our patients are more informed and ask for more comprehensive solutions to their problems. You cannot underestimate the power of patients seeing their own teeth, or missing teeth, in full colour on screen.

'With our on-site digital lab we take these scans to produce digital imagery of a range of possible treatment outcomes using different techniques. Patients no longer have to trust the dentist. They trust their own eyes, both in terms of the problems they see, and the solutions they are presented with.'

This would explain the increase in revenue, but the second, and arguably most impressive fact is that Marcos has not taken on any additional personnel in the last 12 months.

'That was what blew our minds when we were looking at the figures. Not only have we been busier with more comprehensive work - but we've been more efficient in delivering it.'

Marcos' experience with different scanning systems and his constant drive to improve and innovate, led him to building his own on-site digital lab. This fabricates all his implant, veneer and restorative work for his entire practice, and for some other digital dentists across the country.

'It just grew', he says. 'Wed meet like-minded digital dentists who were struggling to get their labs to push forward digitally and we'd offer to lend a hand. Because of our unique perspective of clinical, lab and digital we can mentor dentists with their workflows, and troubleshoot all the common issues they come up against.'

Whilst the scanners are like iPhones in terms of their usability, Marcos explains, everything else is brand new.

'The surprise for many dentists is that they think they can continue with their normal style of dentistry and just scan it. But to get true value and efficiency, you've got to rebuild your skills from the ground up. Re-learn.'

Marcos now runs a series of courses that peel back the curtain on all these aspects - digital consults, consent, digital treatment planning. He deconstructs veneer, implant and Invisalign appointment workflows and shows how you can halve the length, and sometimes the number of appointments.

Previous course delegates comment most frequently that the efficiency this digital workflow brings about is staggering.

Jayme Adams, Dentist for 360 Dental Care, commented: 'Thank you for putting on a fantastic day. We both really enjoyed it and have lots of ideas we are going to implement straight away. We were both extremely impressed with your team, systems and place. Also, we would like to thank you for your generosity in sharing your secrets to success. It's rare to find such abundant attitudes! I genuinely feel the day we spent is going to make a significant impact to our business!'

Marcos' courses can be booked online at digitaldentalschool.co.uk and are run from his practice and training facility in Yorkshire. Delegates are able to see live patients being treated, together with step by step protocols on how to replicate the results. Continuing the contemporary approach, videos and case slides are accessible through the course Whatsapp group which facilitates ongoing mentoring.

Marcos concludes: 'We felt we had to provide this training. The requests we had to share our knowledge were voracious, and for the most part it's people who already have scanners but who don't know how to utilise the full power of the tool they've got who are keenest to attend!'

For more information about joining the course, email Marcos at info@ digitaldentalschool.co.uk so that you can plan to be ahead of the game. After all, the march of digital technology is inevitable!

Visit www.itero.com or www.facebook.com/iterodigitalimpressionsystem/ for the latest news on the iTero Element scanner portfolio.

\footnotetext{
About iTero

The iTero Element ${ }^{\circledR}$ intraoral scanner is a dental chairside platform with a range of integral digital tools and desktop software, developed to enhance accuracy of records, treatment efficiency, and overall patient experience compared to conventional impressions. www.itero.com.
} 